\title{
From the Editors
}

Simone de Beauvoir was in her $60 \mathrm{~s}$ when she wrote this eloquent description of what aging means from the elders' perspective:

... we hardly think about our age any more: we feel that the notion does not apply to us; for it is one which assumes that we look back toward the past and draw a line under the total, whereas in fact we are reaching out toward the future, gliding on imperceptibly from day to day, from year to year. Old age is particularly difficult to assume because we have always regarded it as something alien, a foreign species: 'Can I have become a different being while I still remain myself?'

de Beauvoir's observations are particularly insightful as she points to the lack of correspondence that exists between the experience of aging and the perception of age. We have a preconceived common notion of what old age is, and it is often thought of in pejorative terms, "used up," "infirm," "nonessential." The elderly often say, "I don't feel 75," and this is a reflection of the erroneous expectation of what it means to be old.

Similar assumptions about aging are also found within a medical context, where youth is equated with health, and aging with deterioration. More accurately, youth may be a measure of vigor, strength, and endurance, but not necessarily of health. The athlete who collapses and dies on the basketball court cannot have been healthy, even though his athletic skills exceeded those of most of his peers. Also, we are learning that so-called degenerative diseases of aging are more likely reflections of poor health practices in earlier years, genetics, and environmental factors, than they are inevitabilities of age.

These misconceptions aside, de Beauvoir's description of old age as "something alien, a foreign species" goes a long way in explaining society's response to the elderly. We usually regard whatever is considered "alien" with suspicion, guardedness, and even some hostility. This distancing compromises our ability to identify with the "other" and thereby empathize. It is the objectification of the elderly that creates what Martin Buber called "I-it" relationships where the other remains external to ourselves. Because a genuine relationship is excluded, instrumental use becomes permitted. The utilitarian belief that the elderly are less valuable than other members of society because their contributions to social goals are less is a leitmotif playing through various movements of the resource allocations debates. It appears most explicitly in some libertarian proposals that the elderly as a class should be taxed to take care of themselves. As public policy such an approach, neglecting the contributions already made by the elderly to younger generations, would officially separate them like social lepers.

Perhaps an inescapable aspect of aging is that it is a disquieting reminder 
of our own mortality. How much more comfortable it is to distance ourselves from death by embracing it as a natural event for the elderly but not for ourselves. The acceptability of mortality for the elderly is inversely proportional to its unacceptability for ourselves, leading us once again to avoid those who remind us of what we want to forget.

It is easy to see how this attitude skews our ethical judgments. The more removed we feel from another being, the less sympathetic we tend to be. As a result, we become infected with a xenophobia with regard to old age and the elderly, from which even elders themselves do not escape.

A Harris Poll conducted for the National Council on Aging in the United States discovered that "It is not the young alone who have negative expectations of old age. The older public themselves have bought the stereotypes and myths of old age, and, recognizing that life is not so terrible for them consider themselves the exception to the rule."

The bifurcation between how the healthy elderly feel and how they per- ceive themselves occurs because the effect of society's view is pernicious. Serious consideration needs to be given to whether the alienation and stigmatization of the elderly, common to western cultures, might influence elders to make judgments about the value of their lives negatively and, if so, what this means for medical decision making as well as advance directives.

Therefore, when we explore the ethics of aging in the Special Section on "Elder Ethics," we mean much more than withholding or withdrawing treatment, nursing home placement, or research on Alzheimer's disease. We have in mind an analysis of our personal and social assumptions of aging for the purpose of reaching a deeper understanding of the prevailing ethical xenophobia regarding the elderly and its influence on our moral judgments. Perhaps the ethical myopia that leads people to be unaware of inappropriate value judgments they make regarding old age is not unlike the shortsightedness that causes people who live in a violent society to be blinded to the level of that violence. 